http://doi.org/10.1061/(ASCE)GM.1943-5622.0000244

\title{
Probabilistic Analysis of Soil Consolidation via Prefabricated Vertical Drains
}

\author{
Md. Wasiul Bari ${ }^{1}$, Mohamed A. Shahin ${ }^{2}$ and Hamid R. Nikraz ${ }^{3}$ \\ ${ }^{1} \mathrm{PhD}$ Candidate, Department of Civil Engineering, Curtin University, WA 6845, Australia \\ ${ }^{2}$ Associate Professor, Department of Civil Engineering, Curtin University, WA 6845, Australia \\ ${ }^{3}$ Professor, Department of Civil Engineering, Curtin University, WA 6845, Australia
}

\begin{abstract}
Soil consolidation by prefabricated vertical drains (PVDs) relies on some soil properties that are spatially variable such as the coefficient of permeability. However, the design of soil consolidation via prefabricated vertical drains (PVDs) has been traditionally carried out deterministically and thus can be misleading due to the ignorance of the uncertainty associated with the inherent spatial variation of soil properties. In this technical note, the effects of spatial variability of soil permeability on soil consolidation by PVDs are investigated stochastically, and the corresponding uncertainty associated with the degree of consolidation is statistically quantified and analysed.
\end{abstract}

Keywords: Finite elements; Spatial variability; Consolidation; Vertical drains; Probability. 


\section{Introduction}

Prefabricated vertical drains (PVDs) in combination with pre-loading are a popular technique that is used to enhance the bearing capacity of soft soil by accelerating soil consolidation. Over the years, the analysis of soil consolidation by PVDs has evolved from the approximate theoretical calculations to the advanced numerical solutions. Traditionally, in order to predict soil consolidation, it is often assumed that the consolidating soil surrounding the PVDs is homogeneous. In reality, however, soils are seldom homogeneous but rather spatially variable in the ground and potentially induce uncertainty in their characterization (Vanmarcke 1984). For instance, it was demonstrated by Chang (1985) that the values of coefficient of consolidation can have a fairly degree of variation even in a uniform clay layer. Therefore, investigating the impact of soil spatial variability in treatment of soft soils by PVDs is important in providing an improved conceptual understanding of the soil consolidation problem. However, to the authors' best knowledge, the issue of soil spatial variability has never been previously considered in a systematic, scientific manner in design of soil improvement via PVDs, and the work presented herein is a step forward towards filling in this gap. To date, the majority of probabilistic studies in relation to soil consolidation have been limited to vertical drainage (i.e. no PVDs), and only a few studies (e.g. Hong and Shang 1998; Huang et al. 2010) have focussed on the horizontal drainage by vertical drains. However, in all previous studies, the uncertainty associated with the measurement errors of soil testing was the only factor that has been investigated, and due to the analytical and numerical complexities, inherent soil spatial variability was not considered.

\section{Stochastic Solution of Soil Consolidation by Prefabricated Vertical Drains}

Among several available methods to model stochastic problems in geotechnical engineering, the use of deterministic finite element analysis with stochastic input soil parameters in a 
Monte Carlo framework has gained much popularity in recent years (Elkateb et al. 2002). Similar approach is adopted herein to investigate the effects of soil spatial variability on the behaviour of soil consolidation by PVDs. The different steps taken in the current approach as well as the analytical and numerical techniques used are described in detail below.

\section{Characterization and Generation of Spatially Variable Soil Fields}

Among all soil properties, the spatial variability of soil permeability, $k$, and coefficient of volume compressibility, $m_{v}$, play the most crucial roles in soil consolidation, as indicated by several researchers (e.g. Pyrah 1996). However, in the current study, only $k$ is treated as a random variable and $m_{v}$ is assumed to be constant across the soil mass. This is because as reported by Baecher and Christian (2003), $k$ possesses spatial variability of as high as $300 \%$ compared to only $25-30 \%$ for $m_{v}$, thus, $k$ provides the most significant impact on soil consolidation. The well resistance and smear effect are another influence factors that affect the rate of consolidation (Basu and Prezzi 2007). However, for simplicity, the smear and well resistance are not considered in the current study as they are left for future refinement.

In this study, 2D random permeability field of $k$ is generated using the local average subdivision (LAS) technique developed by Fenton and Vanmarcke (1990), which simplifies the task of generating finite elements from the random field. The permeability field is assumed to be isotropic and thus has equal permeability values in the horizontal and vertical directions (i.e. $k_{h}=k_{v}=k$ ). The permeability $k$ is taken to be constant within each element of the soil domain and its value is given by the local geometric average of the permeability field over the element domain. From element to element, the value of $k$ is varied, reflecting the random nature of the permeability. The spatial variability of $k$ is characterised stochastically by assuming that $k$ is represented by a lognormal distribution that is defined by a mean value, $\mu_{k}$, and standard deviation, $\sigma_{k}$. The lognormally distributed permeability field $k_{i}$ of the $i$ th element is obtained using the following transformation function (Fenton and Griffiths 2008): 


$$
k_{i}=\exp \left\{\mu_{\ln k}+\sigma_{\ln k} g_{\ln k(i)}\right\}
$$

where: $\mu_{\ln k}$ and $\sigma_{\ln k}$ are, respectively, the mean and standard deviation of the logarithm of $k$, and $g_{\operatorname{lnk}(i)}$ is the local (arithmetic) average random field over the domain of the $i$ th element, which is generated from a sequence of standard normally distributed random field $g_{\ln k}$ having zero mean, unit variance and certain spatial correlation. The spatial correlation is estimated mathematically using an isotropic exponentially decaying (Markovian) spatial correlation function, as follows (Fenton and Griffiths 2008):

$$
\rho_{\ln k}(\tau)=\exp \left(-\frac{2|\tau|}{\theta_{\ln k}}\right)
$$

where: $|\tau|$ is the absolute distance between two points in the soil domain and $\theta_{\ln k}$ is the spatial correlation length (or scale of fluctuation) of the underlying normally distributed permeability. It should be noted that $\theta_{\ln k}$ is the distance within which soil is significantly correlated, thus, a large value of $\theta_{\ln k}$ indicates a smoothly varying (homogeneous) field, whereas a small value of $\theta_{\ln k}$ implies an erratic (heterogeneous) field. It should also be noted that, for simplicity, the correlation lengths in the vertical and horizontal directions of the 2D random field generated in this study are taken to be equal (i.e. isotropic).

\section{Finite Element Modelling Incorporating Spatial Variability of Soil Permeability}

In this study, all numerical analyses are carried out using the finite element computer program AFENA (Carter and Balaam 1995). The consolidation process of soil in AFENA is treated as a coupled transient problem governed by the Biot's consolidation theory (Biot 1941). In ground improvement by PVDs, soil consolidation takes place by simultaneous vertical and horizontal (radial) drainage of water. However, as the drainage length in the vertical direction is significantly larger than that of the horizontal direction and permeability in the horizontal 
direction is often much higher than that of the vertical direction (Hansbo 1981), soil consolidation due to vertical drainage is much less than that of the horizontal drainage. Under this reasoning, only soil consolidation due to horizontal drainage is analysed in the current study. The consolidation problem considered in this study implies an axisymmetric finite element simulation of the following geometry (see Fig. 1): $L=1.0 \mathrm{~m}, r_{e}=1.025 \mathrm{~m}$ and $r_{w}=$ $0.025 \mathrm{~m}$, where $L$ is the thickness of consolidating soil layer; $r_{e}$ is the radius of equivalent soil cylinder with impermeable perimeter (or the radius of zone of influence); and $r_{w}$ is the equivalent radius of the drain having a width $b=75 \mathrm{~mm}$ and thickness $a=3 \mathrm{~mm}\left[r_{w}=(a+\right.$ b) $/ \pi]$.

As the accuracy of the finite element analysis is dependent on the mesh density, a sensitivity analysis is carried out for both the deterministic and stochastic solutions on various mesh dimensions to ensure reasonable refinement with minimal discretization error and to produce reliable and reproducible statistics of the output quantities. The sensitivity analysis indicated that a mesh with at least $10 \times 10$ elements is needed in order to obtain a reasonable precision. Based on this observation and in order to comply with the minimum correlation length used, it was decided to discretize the problem into a more refined mesh of $16 \times 16$ elements. The soil skeleton is modelled as linear elastic material with spatially variable $k$ and a constant $m_{v}=7.43 \times 10^{-5} \mathrm{~m}^{2} / \mathrm{kN}$ across the soil mass. The applied drainage and deformation boundary conditions are shown in Fig. 1.

The effects of spatial variability of $k$ on the degree of soil consolidation are investigated using a mean value $\mu_{k}=10^{-9} \mathrm{~m} / \mathrm{sec}$, and for the interest of generality, $\sigma_{k}$ and $\theta_{\ln k}$ are presented in the following normalized forms: $v=\sigma_{k} / \mu_{k}$ (i.e. coefficient of variation) and $\Theta$ $=\theta_{\ln k} / r_{e}$ (i.e. dimensionless scale of fluctuation). The following values of $v$ and $\Theta$ are considered: $v(\%)=25,50,100,200$ and 300 , whereas $\Theta=0.061,0.244,0.488,0.976,1.95$, 3.9 and 7.8 (corresponding to $\theta_{\ln k}(\mathrm{~m})=0.0625,0.25,0.5,1.0,2.0,4.0$ and 8.0). A single 
generation of a random permeability field and the subsequent finite-element analysis of that field are termed "realization". For an individual realization, the degree of consolidation, $U$, at any certain consolidation time, $t$, is calculated in terms of the excess pore water pressure with the help of the following expression:

$$
U=1-\frac{\bar{u}}{u_{0}}
$$

where: $\bar{u}$ and $u_{0}$ are the initial uniform and average excess pore water pressures, respectively.

\section{Repetition of Process Based on the Monte Carlo Technique}

Following the Monte Carlo technique, it is found that 2000 realizations are sufficient to obtain reasonably stable output statistics for each analysis of interest. Using each combination of the statistical parameters $v$ and $\Theta$ mentioned earlier, a series of 35 stochastic analyses are carried out, and the obtained outputs are collated and statistically analyzed to produce estimates of the probability density functions and the corresponding probability events for the degree of consolidation.

\section{Probabilistic Interpretation}

One of the main objectives of the stochastic analyses is to estimate the probability that a deterministic degree of consolidation overestimates the true consolidation value. At any given time, this involves the selection of a reasonable probability distribution for the obtained degree of consolidation data. In order to obtain a reasonable distribution, the degree of consolidation data are transformed into $U^{*}$, which is an alternative representing form for the degree of consolidation $U . U^{*}$ is used herein because it better facilitates the selection of a reasonable probability distribution for the obtained degree of consolidation data. $U^{*}$ is derived from the analytical solution of soil consolidation proposed by Hansbo (1981) in which the degree of consolidation due to the horizontal drainage at time $t$ is obtained by: 


$$
U=1-\exp \left(-\frac{2 k t}{r_{e}^{2} \alpha m_{v} \gamma_{w}}\right)
$$

where: $\gamma_{w}$ is the unit weight of water; $t$ is the consolidation time; $\alpha$ is a group parameter representing the smear effect and geometry of the PVD system. Without considering the effects of smear and well resistance, $\alpha$ can be calculated as follows:

$$
\alpha \approx \ln (n)-0.75
$$

where: $n=r_{e} / r_{w}$ is the drain spacing ratio. The value of $U^{*}$ can thus be obtained by rearranging and rewriting Eq. (4) to yield:

$$
\ln \left[\frac{1}{1-U}\right]=\frac{2 t}{r_{e}^{2} \alpha m_{v} \gamma_{w}} k
$$

Assuming that $\ln [1 /(1-U)]=U^{*}$, Eq. (6) becomes:

$$
U^{*}=\frac{2 t}{r_{e}^{2} \alpha m_{v} \gamma_{w}} k
$$

$U^{*}$ can be considered as the output of a single averaged permeability over a certain individual realization, and if the average permeability over that realization is denoted as $\bar{k}$, Eq. (7) can then be rewritten as follows:

$$
U^{*}=\frac{2 t}{r_{e}^{2} \alpha m_{v} \gamma_{w}} \bar{k}
$$

Since $k$ is lognormally distributed, its average $\bar{k}$ will also be approximately lognormally distributed (over the suite of Monte Carlo simulations). Although the true distribution of $\bar{k}$ may be different from that of the lognormal distribution, this distribution is selected because it offers the advantage of simplicity as it is derived from a simple nonlinear transformation of the classical normal distribution. In addition, the geometric average of a random variable tends to have a lognormal distribution by the central limit theorem. Therefore, it is reasonable to assume that $U^{*}$ in Eq. (8) is also lognormally distributed. Based on this derivation, the data of $U$ obtained from each of the 35 stochastic finite element simulation tests conducted in the 
current study are transformed to $U^{*}$, their histograms are plotted and the rationality of their lognormal distribution hypothesis is assessed and found to be successful for almost all cases (the goodness-of-fitness $p$-value never fell below 0.1 ).

By accepting the lognormal distribution as a reasonable fit for $U^{*}$, the statistical moments, $\mu_{U^{*}}$ and $\sigma_{U^{*}}$ that represent the mean and standard deviation of the lognormally distributed $U^{*}$ are calculated for each set of $v$ and $\Theta$ from the suite of 2000 realizations using the method of moments. For $90 \%$ target degree of consolidation $\left(U_{90}\right)$ (i.e. when $\left.U=0.9\right), U^{*}$ $=\ln [1 /(1-0.9)]=2.3026$. Therefore, the probability of getting $U^{*} \geq 2.3026$ will be equivalent to the probability of achieving $U \geq 90 \%$ (i.e. $P\left[U \geq U_{90}\right]$ ) $=P\left[U^{*} \geq 2.3026\right]$. Then $P\left[U \geq U_{90}\right]$ can be estimated as follows:

$$
P\left[U \geq U_{90}\right]=P\left[U^{*} \geq 2.3026\right]=1-\Phi\left(\frac{\ln 2.3026-\mu_{\ln U^{*}}}{\sigma_{\ln U^{*}}}\right)
$$

where: $P[$.$] is the probability of its argument; \Phi($.$) is the standard normal cumulative$ distribution function; $\mu_{\ln U^{*}}$ and $\sigma_{\ln U^{*}}$ are, respectively, the mean and standard deviation of the underlying normally distributed $\ln U^{*}$ and can be estimated from $\mu_{U^{*}}$ and $\sigma_{U^{*}}$ using the transformation equations between the lognormal and normal distributions.

\section{Results and Discussion}

Although a series of 35 stochastic finite element simulation tests for various combinations of the input statistical parameters are performed, for brevity and limited space, only a few tests are presented in Figs. 2-6. As the probability of achieving 90\% degree of consolidation is estimated on the basis of $U^{*}, \mu_{U^{*}}$ and $\sigma_{U^{*}}$ are presented in Figs. 2-5 versus the consolidation time, $t$. It should be noted that prior to the probabilistic analyses, an initial deterministic analysis of the considered consolidation problem is performed, and the time corresponding to $90 \%$ consolidation of the deterministic solution $\left(t_{\mathrm{D} 90}\right)$ is found to be 31.0 days. 
The effect of $v$ on $\mu_{U^{*}}$ for a constant value of $\Theta=1.95$ (corresponding to $\theta_{\ln k}=2 \mathrm{~m}$ ) is shown in Fig. 2, which also includes the deterministic solution of no soil variability. It can be seen that $\mu_{U^{*}}$ tends toward the deterministic solution for lower values of $v$ (i.e. when soil is becoming more homogeneous), whereas it tends to move away from the deterministic result as $v$ increases (i.e. when soil is becoming more heterogeneous). This implies that more consolidation time is needed for stabilization of heterogeneous soil than homogeneous soil. This is expected due to the fact that less permeable zones of soil are more frequently encountered in heterogeneous soil and thus water flow becomes slower. Similar behaviour is obtained for the other values of $\Theta$. The effect of $v$ on $\sigma_{U^{*}}$ is shown in Fig. 3 for a constant value of $\Theta=1.95$. It can be seen that at any particular consolidation time $t, \sigma_{U^{*}}$ increases with the increase of $v$.

The effect of $\Theta$ on both $\mu_{U^{*}}$ and $\sigma_{U^{*}}$ are illustrated in Figs. 4 and 5 for $v=100 \%$. It can be seen from Fig. 4 that as $\Theta$ increases, $\mu_{U^{*}}$ approaches the deterministic result, which is expected because higher value of $\Theta$ means smoothly varying (uniform) random field of soil. By comparing Figs. 2 and 4, it can be seen that $\mu_{U^{*}}$ is relatively less sensitive to $\Theta$ than $v$. In Fig. 5, it can be seen that at any particular consolidation time $t, \sigma_{U^{*}}$ increases with the increase in $\Theta$. This is because in statistics the variance of an average decreases linearly with the number of independent samples used in the average (Fenton and Griffiths 2008). In the context of random field, the "effective" number of independent samples increases as the correlation length decreases, thus, low variance in the degree of consolidation is to be expected. Conversely, when the correlation length increases, the variance of the degree of consolidation is expected to increase. It is interesting to see that the closely grouped curves corresponding to $\Theta=1.95,3.9$ and 7.8 clearly demonstrate that the increasing rate of $\sigma_{U^{*}}$ gradually decreases with the increase of $\Theta \geq 1.95$. 
As mentioned earlier, one of the main objectives of the stochastic analyses carried out in this study is to enable estimates of the probability of occurrence of certain degree of consolidation events. This is important as it helps the designers to make informed decision regarding the level risk associated with the design. The effect of consolidation time on $P[U \geq$ $U_{90}$ ] for different $v$ at a fixed value of $\Theta=0.488$ (i.e. $\theta_{\ln k}=0.5 \mathrm{~m}$ ) is investigated in Fig. 6 . The deterministic time of achieving $90 \%$ consolidation, $t_{\mathrm{D} 90}$, is also shown in Fig. 6 by the vertical solid line. It can be seen that, for all curves, $P\left[U \geq U_{90}\right]$ increases with the increase of the consolidation time, as expected. More importantly, it can also be seen that at any certain consolidation time $t, P\left[U \geq U_{90}\right]$ decreases with the increase of $v$, implying that the probability of achieving $90 \%$ target degree of consolidation decreases with the increase of permeability variance. However, opposite trends are obtained at the early stage of consolidation of about $t$ $\leq 20$ days in which $P\left[U \geq U_{90}\right]$ increases as $v$ increases. This is expected in statistics because the range of values of $U^{*}$ (or $U$ ) over which the frequency density curve is distributed increases as $v$ increases. In other words, $U^{*}$ distribution "bunches up" at low $v$ and rapidly excludes the area to the right of the stationary target value of $U^{*}=2.3026$. It is also interesting to see that the deterministic solution yields $P\left[U \geq U_{90}\right]$ of less than $50 \%$ for all cases of $v$, implying that there is $50 \%$ risk that soil consolidation will not be achieved within the specified (deterministic) time frame.

\section{Conclusions}

This paper has used the random field theory and finite element modelling to investigate the influence of spatial variability of soil permeability on soil stabilization by prefabricated vertical drains. Results of the study show that the mean and standard deviation of the degree of consolidation are highly sensitive to the variance of soil permeability and less sensitive to the scale of fluctuation. Increasing the permeability variance decreases the mean of degree of 
soil consolidation but increases its variance. On the other hand, increasing the scale of fluctuation generally increases the mean and standard deviation of the degree of consolidation. The probability of achieving $90 \%$ consolidation at a consolidation time corresponding to the deterministically predicted $90 \%$ consolidation time is always less than $50 \%$ over the range of the statistical parameters considered. This result is reassured from the design viewpoint because it indicates that the traditional (deterministic) approach of design of soil consolidation by PVDs may lead to misleading estimates of true degree of consolidation, especially for erratic soils. The results obtained from this research clearly demonstrate the potential of using the stochastic analyses in providing valuable insights into the effects of soil spatial variability on soil consolidation by PVDs and urges the use of probabilistic techniques in routine design practice.

\section{Notation}

The following symbols are used in this paper:

$$
\begin{aligned}
& g_{\ln k}=\quad \text { standard Gaussian random field of normally distributed permeability; } \\
& \bar{g}_{\ln k}=\quad \text { arithmetic average of } g_{\ln k} \text { over a realization; } \\
& g_{\ln k}(i)=\quad \text { local average of normal random field of normally distributed permeability over } \\
& \text { the domain of the } i \text { th element; } \\
& i, n_{\text {sim }}=\quad \text { element number or simulation number; and total number of simulations; } \\
& \bar{k}, k_{i}=\quad \text { average permeability over a realization; and permeability of the } i \text { th element; } \\
& t, t_{\mathrm{D} 90}=\quad \text { consolidation time; and time for deterministic } 90 \% \text { consolidation; } \\
& u_{0}, \bar{u}=\quad \text { initial excess pore water pressure; and average excess pore water pressure; } \\
& U, U_{90}=\quad \text { average degree of consolidation; and 90\% target degree of consolidation; } \\
& U^{*}=\quad \text { monotonic function of } U(\ln [1 /(1-U)]) \text {; } \\
& v=\quad \text { coefficient of variation of permeability; }
\end{aligned}
$$




$$
\begin{aligned}
& \theta_{\ln k}, \Theta=\quad \text { scale of fluctuation of } \ln k \text {; and dimensionless scale of fluctuation; } \\
& \mu_{k}, \mu_{\ln k}=\quad \text { mean of the lognormally and underlying normally distributed } k \text {; } \\
& \mu_{U^{*},} \mu_{\ln U^{*}}=\text { mean of the lognormally and underlying normally distributed } U^{*} \text {; } \\
& \sigma_{k}, \sigma_{\ln k}=\quad \text { standard deviation of the lognormally and underlying normally distributed } \\
& \text { permeability; } \\
& \sigma_{U^{*}}, \sigma_{\ln U^{*}}=\quad \text { standard deviation of the lognormally \& underlying normally distributed } U^{*} \text {; } \\
& \rho_{\ln k}(\tau)=\quad \text { correlation function giving correlation between two points in the } \ln k \text { field; } \\
& \tau=\quad \text { absolute distance between two points in the soil domain; } \\
& P(.), \Phi(.)=\text { probability of its argument; and standard normal cumulative function. }
\end{aligned}
$$




\section{References}

Baecher, G. B., and Christian, J. T. (2003). Reliability and statistics in geotechnical engineering, John Wiley \& Sons, West Sussex, England.

Basu, D., and Prezzi, M. (2007). "Effect of the smear and transition zones around prefabricated vertical drains installed in a triangular pattern on the rate of soil consolidation." International Journal of Geomechanics, 7(1), 34-43.

Biot, M. A. (1941). "General theory of three-dimensional consolidation." Journal of Applied Physics, 12, 155-164.

Carter, J. P., and Balaam, N. P. (1995). "Program AFENA - A general finite element algorithm: Users' Manual." Centre for Geotechnical Research, University of Sydney.

Chang, C. S. (1985). "Uncertainty of one-dimensional consolidation analysis." Journal of Geotechnical Engineering 111(12), 1411-1424.

Elkateb, T., Chalaturnyk, R., and Robertson, P. K. (2002). "An overview of soil heterogeneity: quantification and implications on geotechnical field problems." Canadian Geotechnical Journal, 40, 1-15.

Fenton, G. A., and Griffiths, D. V. (2008). Risk assessment in geotechnical engineering, John Wiley and Sons, New Jersey.

Fenton, G. A., and Vanmarcke, E. H. (1990). "Simulation of random fields via local average subdivision." Journal of Engineering Mechanics, 116(8), 1733-1749.

Hansbo, S. (1981) "Consolidation of fine-grained soils by prefabricated drains." Proceedings of the 10th International Conference on Soil Mechanics and Foundation Engineering, Stockholm, Sweden, 677-682.

Hong, H. P., and Shang, J. Q. (1998). "Probabilistic analysis of consolidation with prefabricated vertical drains for soil improvement." Canadian Geotechnical Journal, 35(4), 666-677.

Huang, J., Griffiths, D. V., and Fenton, G. A. (2010). "Probabilistic analysis of coupled soil consolidation." Journal of Geotechnical and Geoenvironmental Engineering, 136(3), 417-430.

Pyrah, I. C. (1996). "One-dimensional consolidation of layered soils." Geotechnique, 46(3), 555-560.

Vanmarcke, E. H. (1984). Random fields: analysis and synthesis, The MIT Press, Massachusetts. 


\section{Figure Captions}

Fig. 1. Schematic diagram of soil cylinder with prefabricated vertical drain

Fig. 2. Effect of $v$ on $\mu_{U^{*}}$ for $\Theta=1.95$

Fig. 3. Effect of $v$ on $\sigma_{U^{*}}$ for $\Theta=1.95$

Fig. 4. Effect of $\Theta$ on $\mu_{U^{*}}$ for $v=100 \%$

Fig. 5. Effect of $\Theta$ on $\sigma_{U^{*}}$ for $v=100 \%$

Fig. 6. $P\left[U \geq U_{90}\right]$ versus consolidation time, $t$, for $\Theta=0.488$ (i.e. $\theta_{\ln k}=0.5 \mathrm{~m}$ ) and various v 


\section{Figures for IJOG Technical Note (Revised for Editor)}

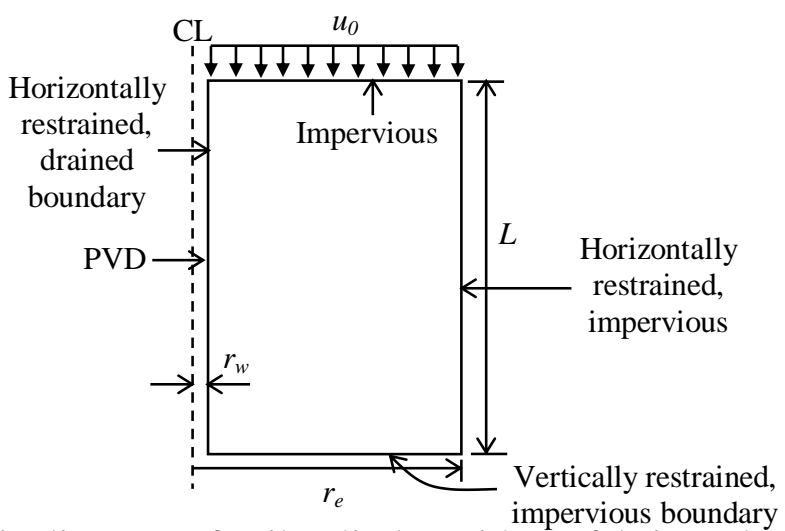

Fig. 1. Schematic diagram of soil cylinder with prefabricated vertical drain

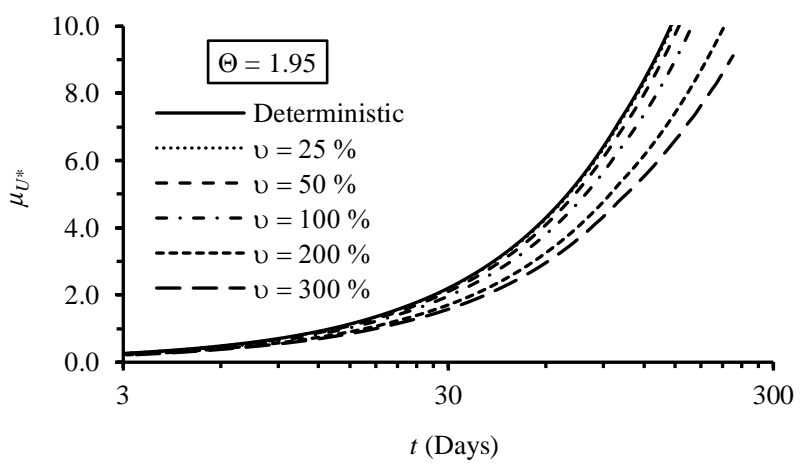

Fig. 2. Effect of $v$ on $\mu_{U^{*}}$ for $\Theta=1.95$ 


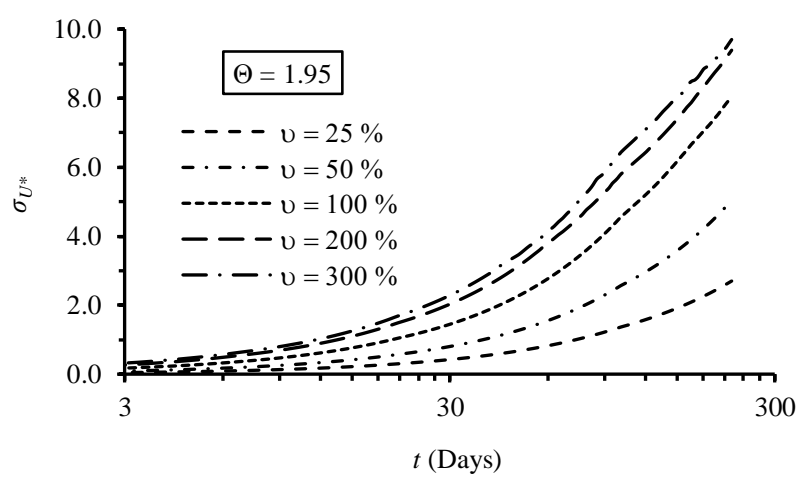

Fig. 3. Effect of $v$ on $\sigma_{U^{*}}$ for $\Theta=1.95$

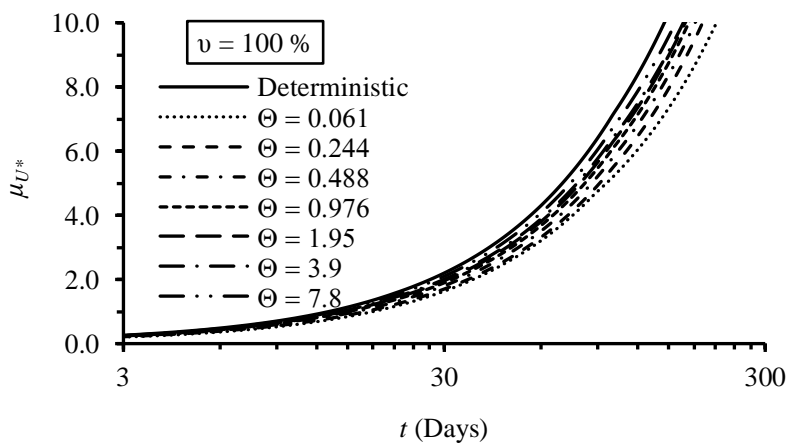

Fig. 4. Effect of $\Theta$ on $\mu_{U^{*}}$ for $v=100 \%$

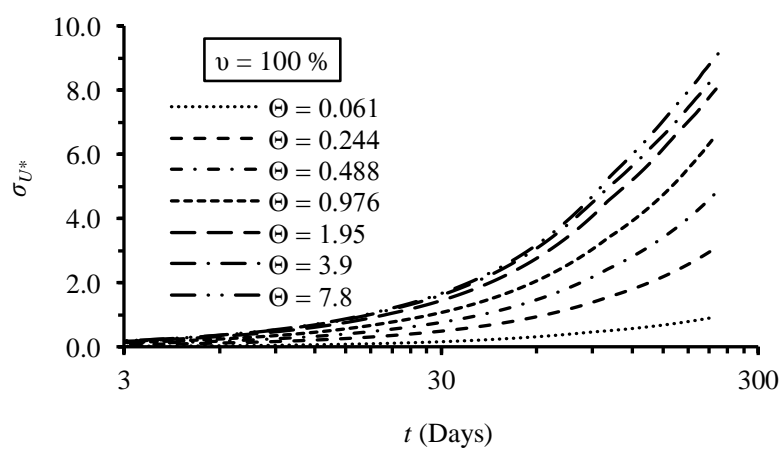

Fig. 5. Effect of $\Theta$ on $\sigma_{U^{*}}$ for $v=100 \%$ 


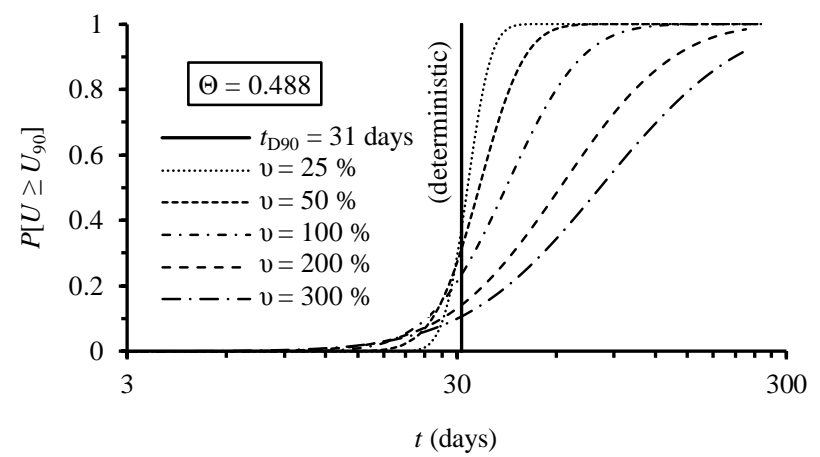

Fig. 6. $P\left[U \geq U_{90}\right]$ versus consolidation time, $t$, for $\Theta=0.488$ (i.e. $\theta_{\ln k}=0.5 \mathrm{~m}$ ) and various $v$ 